\title{
The association between antidepressant treatment and brain connectivity in two double-blind, placebo-controlled clinical trials: a treatment mechanism study
}

\author{
Yun Wang, Joel Bernanke, Bradley S Peterson, Patrick McGrath, Jonathan Stewart, Ying \\ Chen, Seonjoo Lee, Melanie Wall, Vanessa Bastidas, Susie Hong, Bret R Rutherford, David \\ J Hellerstein, Jonathan Posner \\ New York State Psychiatric Institute (Y Wang PhD, P McGrath MD, J Stewart MD, Y Chen MD, S \\ Lee PhD, M Wall PhD, V Bastidas BS, S Hong BS, B R Rutherford MD, D J Hellerstein MD, J \\ Posner MD) and Columbia University College of Physicians and Surgeons ( $Y$ Wang, J Bernanke \\ MD, P McGrath, J Stewart, S Lee, M Wall, B R Rutherford, D J Hellerstein, J Posner), Columbia \\ University, New York, NY, USA; Department of Psychiatry, Keck School of Medicine, Los Angeles, \\ CA, USA (B S Peterson MD); and Institute for the Developing Mind, The Saban Research \\ Institute, Children's Hospital Los Angeles, CA, USA (B S Peterson)
}

\section{Summary}

Background-Antidepressant medications offer an effective treatment for depression, yet nearly $50 \%$ of patients either do not respond or have side-effects rendering them unable to continue the course of treatment. Mechanistic studies might help advance the pharmacology of depression by identifying pathways through which treatments exert their effects. Toward this goal, we aimed to identify the effects of antidepressant treatment on neural connectivity, the relationship with symptom improvement, and to test whether these effects were reproducible across two studies.

\begin{abstract}
Methods-We completed two double-blind, placebo-controlled trials of SNRI antidepressant medications with MRI scans obtained before and after treatment. One was a 10-week trial of duloxetine (30-120 mg daily; mean $92.1 \mathrm{mg} /$ day [SD 30.00]) and the other was a 12-week trial of desvenlafaxine (50-100 mg daily; $93.6 \mathrm{mg} /$ day [16.47]). Participants consisted of adults with persistent depressive disorder. Adjusting for sex and age, we examined the effect of treatment on whole-brain functional connectivity. We also examined correlations between change in functional connectivity and improvement in symptoms of depression (24-item Hamilton Depression Rating Scale) and pain symptom severity (Symptom Checklist-90-Revised).
\end{abstract}

Findings-Participants were enrolled between Jan 26, 2006, and Nov 22, 2011, for the duloxetine RCT and Aug 5, 2012, and Jan 28, 2016, for the desvenlafaxine RCT. Before and after treatment MRI scans were collected in 32 participants for the duloxetine RCT and 34 participants for the desvenlafaxine RCT. In both studies, antidepressants decreased functional connectivity

Correspondence to: Dr Jonathan Posner, Columbia University College of Physicians and Surgeons and New York State Psychiatric Institute, Columbia University, New York, NY 10032, USA, jonathan.posner@ nyspi.columbia.edu.

Contributors

YW, DJH, and JP conceptualised and led the writing of this Article. YC, VB, and SH contributed to the data collection and data extraction. SL and MW contributed to statistical analysis. All authors contributed to interpretation of the data, drafting of the manuscript, approval of the final manuscript, and were responsible for the decision to submit the manuscript. 
compared with placebo (duloxetine study: $\beta=-0.06 ; 95 \% \mathrm{CI}-0.08$ to $-0.03 ; \mathrm{p}<0.0001, \eta_{\mathrm{p}}{ }^{2}=$ 0.44 ; desvenlafaxine study: $-0.06,-0.09$ to $-0.03 ; \mathrm{p}<0.0001, \eta_{\mathrm{p}}{ }^{2}=0.35$ ) within a thalamocortico-periaqueductal network that has previously been associated with the experience of pain. Within the active drug groups, reductions in functional connectivity within this network correlated with improvements in depressive symptom severity in both studies (duloxetine study: $\mathrm{r}=0 \cdot 38$, 95\% CI 0.01-0.65; $\mathrm{p}=0.0426$; desvenlafaxine study: 0.44, 0.10-0.69; $\mathrm{p}=0.0138)$ and pain symptoms in the desvenlafaxine study $(0.39,0.04$ to $0.65 ; \mathrm{p}=0.0299)$.

Interpretation-The findings suggest the thalamo-cortico-periaqueductal network associated with the experience of pain is a new and potentially important target for novel antidepressant therapeutics.

Funding -National Mental Health Institute, Eli Lilly and Company, Pfizer Pharmaceuticals, and the Edwin S Webster Foundation.

\section{Introduction}

Depression is a common disorder with a lifetime prevalence of nearly $20 \% .{ }^{1}$ It has substantial, negative long-term consequences on quality of life, including impaired work performance, disrupted interpersonal relationships, and increased mortality. ${ }^{2,3}$ Although antidepressant medications can provide effective treatment, nearly $50 \%$ of patients either do not respond or have side-effects rendering them unable to continue their course of treatment. ${ }^{4}$ More refined treatments are needed to address this major public health concern, and yet, the past decade has seen a marked slowing in treatment advances.

An important pathway for treatment development is to identify targets for novel interventions by examining not only whether an intervention alleviates depression, but also how this happens, suggesting potential mechanisms by which existing treatments exert their salutary effects. This approach might provide neurobiological targets against which novel treatments can be tested. For example, the experimental medicine initiative of the National Institute of Mental Health replaces clinical improvement with target engagement as the initial outcome of interest for new clinical trials. ${ }^{5}$

Although the effects of treatments on brain structure and function might differ between classes of antidepressants (eg, tricyclic antidepressants $v s$ selective serotonin reuptake inhibitors), common effects should be detectable for different medications within the same class. We reasoned that testing of treatment effects for two different antidepressants, within the same class, should safeguard generalisability within a medication class, curtail spurious or idiosyncratic associations with a particular medication, and provide a robust approach toward identifying reproducibility of biological effects.

In this study, we aimed to identify the effects of antidepressant treatment on neural connectivity and whether these effects were associated with symptom improvement and reproducible across two studies. We coupled resting state functional MRI with placebocontrolled, clinical trials of antidepressants in adults with persistent depressive disorder, with MRI scans obtained before and after treatment. We examined two studies, the first compared duloxetine with placebo ${ }^{6}$ and the second compared desvenlafaxine with placebo; ${ }^{7}$ both 
medications are SNRIs. We used a data-driven, whole-brain, connectomic approach to isolate reproducible treatment effects.

\section{Methods}

\section{Study design and participants}

The duloxetine and desvenlafaxine studies were both randomised controlled trials (RCTs) with double-blind and placebo-controlled designs. ${ }^{6,7}$ In both studies (done at the New York State Psychiatric Institute, New York, NY, USA), subsamples of the participants who enrolled in the RCTs also participated in MRI scanning before and after treatment as other outcome measures, not reported previously. For the 10-week duloxetine study, doses ranged from 30-120 mg daily (mean $92 \cdot 1 \mathrm{mg} /$ day [SD 30.00]). For the 12-week desvenlafaxine study, doses ranged from 50-100 mg daily ( $93.6 \mathrm{mg} /$ day [16.47]). Full descriptions of each RCT are provided elsewhere. ${ }^{6,7}$ Compared with the RCT-only participants, RCT with MRI participants included more men and were younger in age (appendix); we therefore adjusted for sex and age in subsequent analyses.

Both the duloxetine study and the desvenlafaxine study included adults meeting DSM-5 criteria for persistent depressive disorder, subtypes with pure dysthymic syndrome or with intermittent major depressive episodes, without current episode (or dysthymic disorder by DSM-IV). Participants were excluded if they were at high risk of suicide; met criteria for bipolar, psychotic, or substance use disorders, or dementia; or had clinically significant medical illness. Participants with previous psychotropic treatment, or comorbid anxiety disorders remained eligible. Diagnoses were made by clinical interview and confirmed with the Structured Clinical Interview (SCID). ${ }^{8}$ A trained rater assessed depressive symptoms using the 24-item Hamilton Depression Rating Scale (HAM-D). ${ }^{9}$ The Institutional Review Board of the New York State Psychiatric Institute approved the study procedures. Participants provided written informed consent.

\section{MRI pulse sequences}

Images were acquired on a GE-Signa-3T MRI scanner (GE Healthcare, Chicago, IL, USA). Following acquisition of T1-weighted images $(1 \times 1 \times 1 \mathrm{~mm}$ voxel size, $6 \mathrm{~ms}$ repetition time (TR), $2.38 \mathrm{~ms}$ echo time (TE), $11^{\circ}$ flip angle, $256 \mathrm{~mm}$ field of view [FOV], $256 \times 256$ matrix, 166 slices), axial echoplanar images were collected ( $2200 \mathrm{~ms}$ TR, $30 \mathrm{~ms}$ TE, $90^{\circ}$ flip angle, $62.5 \mathrm{kHz}$ receiver bandwidth, single excitation per image, $3.5 \mathrm{~mm}$ slice thickness, 34 slices per volume, $24 \times 24 \mathrm{~cm}$ FOV, $64 \times 64$ matrix), providing an effective resolution of $3.75 \times 3.75 \times 3.5 \mathrm{~mm}$ and whole-brain coverage. Participants were instructed to remain still with their eyes closed and to let their minds wander freely. Two 5-min resting state scans were obtained for each participant. The same imaging procedures were used for the before and after treatment scans.

\section{Image processing}

Image analysis was done with SPM12, CONN toolbox v17.b, and MATLAB. Standard preprocessing procedures included slice-timing and motion correction, segmentation, normalisation to Montreal Neurological Institute space, spatial smoothing $(8 \mathrm{~mm}$ full width 
at the half maximum Gaussian kernel), temporal band-pass filtering (0.008-0.09 Hz), and linear detrending. We controlled for five orthogonal time series and their derivatives from white matter and cerebrospinal fluid using component-based noise reduction. ${ }^{10} \mathrm{We}$ controlled for head motion first through regression of six head motion parameters plus their temporal first derivatives. We also used so-called scrubbing by removing outlier volumes, defined as frame-wise displacement (FD) of more than $0.5 \mathrm{~mm}$ from the previous frame or global mean intensity of more than 3 SDs. If more than 30 volumes were scrubbed (ie, $>20 \%$ of the acquired volumes), we excluded the runs from subsequent analysis (no runs met this cutoff). Mean FD and the number of scrubbed volumes were similar across groups and studies (appendix).

\section{Multi-voxel pattern analysis (MVPA)}

MVPA is a whole-brain connectomic approach within the CONN toolbox and has been well validated. ${ }^{10-13}$ MVPA calculates connectivity between residualised BOLD time series within a single voxel and all other voxels in the brain; it repeats this process voxel-wise across the entire brain. By using principal component analysis (PCA), the high dimensionality of the data is reduced to 64 dimensions (ie, spatial components) within each voxel. MVPA thus creates whole-brain connectivity maps for each participant at each timepoint (ie, before and after treatment). In the MVPA-derived maps, each voxel is populated with 64 factor scores summarising the voxel's connectivity with all other voxels in the brain. Following convention, we retained the first five of these 64 components, ${ }^{11,14,15}$ to yield MVPA-derived maps with each voxel ascribed five values indexing its whole-brain connectivity (the distribution of variance [80-85\%] in whole-brain functional connectivity can be explained by five principal components; appendix).

We then did a repeated-measure multivariate ANCOVA (MANCOVA) with each participant's five factor scores at each voxel (ie, MVPA-derived maps) as the dependent variables and with treatment and time as the two independent variables. Participants from both studies were included in the MANCOVA. Treatment was coded as either active treatment (duloxetine or desvenlafaxine) or placebo, and time was coded as either study baseline (ie, pretreatment) or endpoint (ie, post-treatment). We included RCT (duloxetine vs desvenlafaxine), age, and sex as covariates. The treatment-by-time interaction was isolated. To safeguard that the results were not contingent upon the numbers of retained PCA components, we re-ran the MANCOVA after varying the numbers of retained components from the MVPA and found similar results (appendix).

\section{Network analysis}

We aimed to determine whether the antidepressant treatment-associated clusters identified by MVPA represented connectivity changes within a defined neural network. Visual inspection suggested substantial overlap between our MVPA results and the "neurologic signature of physical pain," a neural network associated with the experience of physical pain (henceforth referred to as the pain network). ${ }^{16}$ To test this observation empirically, we calculated the cosine similarity between our MVPA results and a mask of the pain network provided by the Wager lab and ten other well defined neural networks from Smith and colleagues. $^{17}$ 
We hypothesised that SNRIs cause a reduction in connectivity within the pain network. To test this, we used graph analysis by first extracting residualised BOLD time series from pain network nodes ( $5 \mathrm{~mm}$ radius). To prevent so-called double dipping, we did not derive the pain network nodes or regions of interest (ROIs), from our MANCOVA results, but instead used 16 ROIs and their corresponding stereotactic coordinates, defined elsewhere from independent studies of the pain network. ${ }^{16}$ We correlated these pain network ROIs regionby-region, yielding a $16 \times 16$-correlation matrix per participant. To maintain consistency with previous analyses on duloxetine's effects on network connectivity, ${ }^{18}$ we used network density as the graph theory metric to quantify network connectivity and calculated network density with UCINET. Finally, for each study, we did a separate ANCOVA with before versus after treatment change in pain network density as the dependent variable, with treatment and time as independent variables, and with age and sex as covariates. We also examined the two studies together and assessed treatment-by-time-by-RCT interactions. In addition to our network-based analysis, we did an ROI-to-ROI analysis (appendix).

\section{Clinical correlates}

Adjusting for sex and age, we examined partial correlations between change in pain network density and improvement in pain symptom severity derived from the Symptom Checklist-90Revised (SCL-90-R; appendix). Using the same covariates, we examined partial correlations between change in pain network density and improvement in depressive symptoms (before $v s$ after treatment HAM-D summary score). We calculated the partial correlations and $95 \%$ CIs in each study separately. We explored non-parametric and non-linear associations using spearman rank partial correlations and multivariate regression analysis with and without non-linear terms, respectively (appendix).

\section{Mediation analysis}

We examined the role that the pain network might have in the causal pathway from treatment ( 1 for active drug and 0 for placebo) to outcome (ie, symptom improvement; before $v s$ after treatment HAM-D summary score). Taking change in pain network density to be the potential mediator, we used modern causal methods for decomposing the total treatment effect into mediation and interaction effects. ${ }^{19}$ Baseline measures of pain network density, baseline depression symptom severity, sex, and age were added as covariates in both the model predicting the mediator and the model predicting the outcome. Within the counterfactual causal mediation framework, ${ }^{20-22}$ interactions between treatment and mediator should also be considered. This framework allows the total effect to be decomposed into four components: (1) controlled direct effect (CDE) indicating how treatment affects outcome without involving the mediator; (2) reference interaction (or $\mathrm{INT}_{\text {ref }}$ ), which indicates how changes in the mediator affect the outcome when the exposure is not present (ie, in the placebo arm); (3) mediated interaction (or $\mathrm{INT}_{\text {med }}$ ), which indicates how change in the mediator is itself necessary for the drug to affect the outcome, and (4) pure indirect effect (PIE), which indicates how treatment affects the outcome purely through its ability to change the mediator. We examined this decomposition (including treatment $x$ mediator interaction) separately for each study, as well as together. Since the mediator (pain network) and outcome (HAM-D symptom score) were observed without temporal separation, we cannot rule out the hypothesis that change in the depressive symptoms is the 
mediator and pain network density is the outcome. Hence, we also did the same mediation analyses but switched the roles of mediator and outcome.

\section{Reproducibility}

To facilitate reproducibility of our approach, we have provided all data analytic code on github.

\section{Role of the funding source}

Funders of the study had no role in study design, data collection, data analysis, data interpretation, or writing of the report. YW, DJH, and JP had full access to all the data in the study and take responsibility for the integrity of the data and the accuracy of the data analysis. All authors made the decision to submit for publication.

\section{Results}

Between Jan 26, 2006, and Nov 22, 2011, the duloxetine RCT enrolled 65 participants in total, of whom 51 consented to participate in the RCT with MRI scans (appendix). 41 completed the pretreatment MRI scan and 32 completed both scans (appendix). Between Aug 5, 2012, and Jan 28, 2016, the desvenlafaxine RCT enrolled 59 participants, with a subsample of 42 consenting to participate in the RTC with MRI scans (appendix). 34 participants completed both MRI scans (appendix). Both RCTs showed significant effects of active treatment over placebo on depressive symptoms (appendix). Completers versus dropouts had similar demographics and clinical measures (table 1, appendix).

MANCOVA of the MVPA-derived maps revealed 18 clusters with a significant treatmentby-time interaction (figure 1 , table $2, \mathrm{p}_{\mathrm{fdr}}<0.05$ ). Similar results were found when we ran the duloxetine and desvenlafaxine studies separately, and for both studies combined (appendix). We also converted the map of the MVPA results to an effect size map with medium to large effect sizes (appendix). Across all of the networks examined, our MVPA results showed the greatest similarity with the pain network (appendix).

In the duloxetine study, pain network density decreased in the active treatment group compared with placebo $\left(\beta=-0.06 ; 95 \%\right.$ CI -0.08 to $-0.03 ; \mathrm{p}<0.0001, \eta_{\mathrm{p}}{ }^{2}=0.44$; figure 2). Similarly, in the desvenlafaxine study, active treatment decreased the density of the pain network compared with placebo $\left(-0.06,-0.09\right.$ to $-0.03 ; \mathrm{p}<0.0001, \eta_{\mathrm{p}}{ }^{2}=0.35$; figure 2$)$. When we pooled the two studies, the effect of the active treatment did not differ by study (treatment $\times$ study interaction, $-0.005,-0.05$ to $0.04 ; p=0.80, \eta_{p}{ }^{2}=0.0011$ ). Before treatment, pain network density did not differ between placebo and active treatment groups in either study separately (duloxetine study: mean difference of $0.01,95 \% \mathrm{CI}-0.02$ to 0.05 ; $\mathrm{p}=0.52$; desvenlafaxine study: $-0.04,-0.08$ to $0.00 ; \mathrm{p}=0.0703)$ or together $(0.02,-0.01$ to $0 \cdot 04 ; \mathrm{p}=0 \cdot 29$ ). Examining the network results with a binary classification (ie, increase $v s$ decrease in network density) yielded similar results (appendix). Test-retest reliability for the measurement of pain network density (measured before and after treatment) was good (appendix). After FDR correction, treatment effects were not significant for any of the ROIto-ROI connections across the 16 nodes of the pain network (appendix). 
In addition to nodes within the pain network (*; table 2), six other nodes had a significant treatment-by-time interaction (table 2). When tested separately, the associations between connectivity change and symptom improvement were not significant for any of these nodes $(\mathrm{p}>0 \cdot 8)$.

After controlling for age and sex, improvement in pain symptoms on the SCL-90-R was associated with change in pain network density in both studies examined separately (duloxetine study: $\mathrm{r}=0.36,95 \%$ CI 0.09 to $0.58 ; \mathrm{p}=0.0111$; desvenlafaxine study: 0.30 , 0.06 to $0.51 ; p=0.0128)$. Improvement in pain symptoms was also associated with decrease in pain network density within the active treatment group of the desvenlafaxine study ( $0 \cdot 39$, 0.04 to $0.65 ; \mathrm{p}=0.0299$; appendix), but not within the active treatment group of the duloxetine study (0.26, $-0 \cdot 19$ to $0 \cdot 62 ; \mathrm{p}=0.26$; appendix). Similar results were detected with Spearman rank partial correlation (appendix), and multivariate regression analysis with a non-linear term and sensitivity analysis (appendix).

We did not detect associations between improvement in pain symptoms and change in pain network density within the placebo group of either study $(\mathrm{p}>0 \cdot 6)$ and the association between pain symptom improvement and pain network change did not differ for active treatment versus placebo in either study $(p>0.4)$. Therefore, we did not explore mediation with pain symptoms as an outcome.

After controlling for age and sex, improvement in symptoms of depression (HAM-D) was not associated with change in pain network density (duloxetine study: $r=0 \cdot 21,95 \%$ CI -0.04 to $0.43 ; p=0 \cdot 11$; desvenlafaxine study: $0 \cdot 18,-0.06$ to $0.41 ; p=0 \cdot 13$ ). However, within the active treatment group only, improvement in HAM-D was associated with decrease in pain network density in both studies examined separately (duloxetine study: $0.38,0.01$ to $0.65 ; \mathrm{p}=0.0426$; desvenlafaxine study: $0.44,0 \cdot 10$ to $0.69 ; \mathrm{p}=0.0138$; figure 3 ). By contrast, we found a worsening of symptoms associated with decrease in pain network density within the placebo group of the duloxetine study $(-0.48,-0.71$ to $-0.16 ; \mathrm{p}$ $=0 \cdot 0046)$, but not in the desvenlafaxine study $(-0 \cdot 22,-0.51$ to $0.12 ; \mathrm{p}=0 \cdot 21$; figure 3$)$. The association between HAM-D improvement and pain network change was greater for active treatment versus placebo in both studies (duloxetine study: fisher's $z=2 \cdot 7,95 \%$ CI $0 \cdot 26$ to 1.40; $p=0.0069$; desvenlafaxine study: $1.8,-0.06$ to $1.17 ; p=0.0711)$. In both studies, the association between HAM-D improvement and pain network change was similar with active treatment $(-0.18,-0.69$ to $0 \cdot 57 ; \mathrm{p}=0.86)$ and with placebo $(-1 \cdot 12,-0.96$ to $0.28 ; \mathrm{p}=0.26)$. Results detected with Spearman rank partial correlation (appendix) and multivariate regression analysis with a non-linear term and sensitivity analysis were similar (appendix).

Given the limited statistical power to examine mediation effects in each study separately, we have also presented the results of the two studies examined together (appendix). With the two studies pooled, we found that active treatment improved symptoms of depression (total effect $6 \cdot 48,95 \%$ CI $2 \cdot 73-10 \cdot 23 ; \mathrm{p}=0 \cdot 0010)$. We found a mediated interaction effect (INT ${ }_{\text {med }} 6 \cdot 94,95 \%$ CI 1.82-12.06; $\mathrm{p}=0.0087$ ), consistent with the hypothesis that reducing pain network density is necessary for active treatment to reduce depressive symptoms. However, we also found a PIE in the opposite direction $(-3 \cdot 92,95 \%$ CI $-7 \cdot 18$ to $-0 \cdot 62 ; \mathrm{p}=$ 0.0187 ), indicating that in the absence of active treatment, reducing pain network density 
might worsen depressive symptoms. CDE and $\mathrm{INT}_{\text {ref }}$ were not significant $(\mathrm{p}=0.1088$ and $\mathrm{p}$ $=0.7527$, respectively. When we reversed mediator and outcome, we again found a significant mediated interaction ( $\mathrm{INT}_{\text {med }}=0.03,95 \%$ CI 0.00-0.05; $\mathrm{p}=0.019$ ); appendix), indicating that improvement in depressive symptom could mediate the change in pain network density seen with active treatment.

\section{Discussion}

In this study of the neurobiological effects of antidepressant treatment from two RCTs, we found that two SNRIs significantly reduced connectivity (as measured by network density) within a thalamo-cortico-periaqueductal network associated with pain. By contrast, the placebo treatment had no effects on the density of this network. Change in pain network density was correlated with improvements in depressive symptoms, but only in the active treatment groups. Findings from these data-driven analyses point to new hypotheses about potential pathways by which antidepressants exert their salutary effects.

In addition to the small existing literature of studies coupling longitudinal functional neuroimaging with randomised, placebo-controlled trials, several studies have used casecontrol designs with patients having neuroimaging done before and after open-label treatment and healthy controls scanned over a similar interval. Case-control studies have shown increased default mode network ${ }^{18,23}$ and frontolimbic connectivity ${ }^{24,25}$ in depression, potentially related to depressive ruminations and impaired emotion regulation, ${ }^{26,27}$ respectively, and suggest that antidepressants might normalise connectivity in these circuits. ${ }^{28}$ Case-control designs, however, are limited by correlational stipulations, such as unaddressed confounds and non-specific placebo effects. Incorporating randomisation and placebo control, our studies directly impute causality, ${ }^{29}$ explicitly attributing the observed change in the brain's functional architecture to an antidepressant.

Our results suggest important associations between the thalamo-cortico-periaqueductal network and antidepressant treatment. Well characterised in task-related functional MRI (fMRI) research, this network has been termed the "neurologic signature of physical pain," or the pain network, and encompasses 16 regions that coactivate during physical pain. ${ }^{16}$ It comprises regions including the periaqueductal gray, insula, secondary somatosensory, cingulate cortices, superior temporal, and supramarginal gyri. ${ }^{16}$ In our graph analysis of these pain network ROIs, ${ }^{16}$ we detected an association between improvement in pain symptoms and reduction in connectivity within this network. However, because our study did not include task-based fMRI data, some caution is warranted in interpreting the functional and behavioural significance of this network.

Depression and physical pain are often comorbid, and can share common neural alterations. ${ }^{30}$ Moreover, SNRI medications are effective in treating not only depression but also pain; treatment with duloxetine attenuates task-related activations to painful stimulation within pain-associated regions ${ }^{31}$ and early improvement in pain symptoms in patients with depression predicts better remission. ${ }^{32}$ Likewise, regions within the pain network might be functionally heterogeneous and several have been associated with depression (eg, cingulate and insula cortices). Additionally, physical pain can lead to depression, particularly when the 
pain is chronic, and conversely, depression can give rise to physical pain, potentially by lowering the pain threshold. ${ }^{33-35}$ Our findings raise important questions about the association between the pain network and antidepressant treatment. For example, do antidepressants other than SNRI medications reduce pain network connectivity, or are subgroups of patients with depression more likely to remit when their treatment alters pain network connectivity. Patients in our study were diagnosed with persistent depressive disorder and might differ from those with episodic major depressive disorder in emotional and pain dysregulation; differentiating these associations could be an area of focus for future studies.

As well as the pain network, our MVPA analysis identified six additional regions in which connectivity changed significantly with treatment, therefore suggesting that antidepressant effects are limited to the pain network would be inaccurate. However, before and after treatment change in connectivity within these regions was not associated with symptom improvement. Similarly, in the duloxetine study, we previously reported that treatment reduces default mode network connectivity, ${ }^{18}$ but this effect was not replicable in the desvenlafaxine study. ${ }^{36}$ This outcome could reflect mechanistic differences between these two medications. The effects of duloxetine treatment on default mode network connectivity were not associated with symptom improvement ${ }^{18}$ and thus could reflect secondary, or compensatory, responses unrelated to its effects on symptom course.

Regarding mediation, our findings were consistent with the hypothesis that antidepressants decrease pain network density, and that this effect facilitates the efficacy of the active drug treatment on depression. However, the association between change within the pain network and improvement in depressive symptoms differed significantly for active treatment versus placebo. Specifically, without presence of active treatment, we observed that reductions in pain network density were related to a worsening of depressive symptoms. Taken together, these findings suggest that reductions in pain network density might not be sufficient to reduce depressive symptoms (otherwise similar effects should have been evident in the placebo group, or PIE in the mediation decomposition). Rather, our findings suggest that reductions in pain network connectivity must be coupled with other drug treatment effects to facilitate symptom improvement. Identifying these additional drug effects will require subsequent research. For example, with stimulation techniques in animal models, testing whether inducing changes in pain network connectivity augments, or facilitates, the effect of other neurophysiological changes on depressive phenotypes might be feasible.

Although our primary mediation model assumed that the pain network mediated drug effect on symptoms of depression, our study cannot empirically impute causal, or directional, associations between the pain network and symptom change. Our findings are equally supportive of two alternative hypotheses: antidepressants reduce pain network density and this effect on the pain network contributes to improvements in depressive symptoms; or antidepressants reduce depressive symptoms and this effect on depressive symptoms contributes to reductions in pain network density. Subsequent research is needed to disentangle these two possibilities. For example, future studies might include an additional MRI scanning session obtained after the initiation of treatment, but before the conclusion of the study, providing temporal separation between mediator and outcome. 
Additional study limitations are important to consider. First, the duloxetine and desvenlafaxine MRI studies both comprised subsamples of participants from the RCTs and these subsamples differed on age and sex from the full samples. Selection bias could thus limit the generalisability of our findings. Unmeasured variables, such as smoking history or menstrual cycle, could further confound study findings. Second, despite randomisation, more men received placebo than active treatment in the duloxetine study. Although we adjusted for sex in our analyses, we cannot entirely exclude sex effects as a potential confound. Third, most study participants had previous exposure to antidepressants, and thus we cannot exclude the possibility that our findings are attributable to this exposure. Antidepressant exposure could prime patients for a response in the pain network upon subsequent exposures to antidepressants. This theory seems unlikely, because if the reduction in pain network density were merely an artifact of previous antidepressant exposure, reduction in pain network density would be unlikely to mediate symptom improvement. Fourth, our graph analysis used network density to summarise connectivity across the pain network. This data reduction technique minimises statistical comparisons (and related type I error), and showed measurement reliability, but across the two RCTs might have obscured subtle differences in treatment effects on the pain network. Finally, although consistent findings were detected across two studies, the sample sizes were small, and thus replication with larger samples is still needed.

In conclusion, our findings suggest the pain network is a novel and potentially important target for new antidepressant therapeutics and thereby offer new areas for research in treatment development.

\section{Supplementary Material}

Refer to Web version on PubMed Central for supplementary material.

\section{Acknowledgments}

This study was supported in part by National Mental Health Institute grants R01-MH101172 and R01-MH036197 (JP), Eli Lilly and Company, Pfizer Pharmaceuticals, and the Edwin S Webster Foundation.

Declaration of interests

PM has received research support from Naurex, F Hoffman-LaRoche. JS has received research support from Pfizer, Alkermes, Forest, and Shire Pharmaceuticals. DJH has received research support from Pfizer Pharmaceuticals, Eli Lilly and Company, Takeda, Assurex, Marinus, Intra-Cellular Therapies, and Compass Pathways. JP has received research support from Shire Pharmaceuticals and Aevi Genomics. All other authors declare no competing interests.

\section{References}

1. Kessler RC, Berglund P, Demler O, et al. The epidemiology of major depressive disorder: results from the National Comorbidity Survey Replication (NCS-R). JAMA 2003; 289: 3095-105. [PubMed: 12813115]

2. Fergusson DM, Woodward LJ. Mental health, educational, and social role outcomes of adolescents with depression. Arch Gen Psychiatry 2002; 59: 225-31. [PubMed: 11879160]

3. Lehtinen V, Joukamaa M. Epidemiology of depression: prevalence, risk factors and treatment situation. Acta Psychiatr Scand Suppl 1994; 377: 7-10. [PubMed: 8053370] 
4. Trivedi MH, Rush AJ, Wisniewski SR, et al. Evaluation of outcomes with citalopram for depression using measurement-based care in STAR* D: implications for clinical practice. Am J Psychiatry 2006; 163: 28-40. [PubMed: 16390886]

5. Insel TR. The NIMH experimental medicine initiative. World Psychiatry 2015; 14: 151-53. [PubMed: 26043323]

6. Hellerstein DJ, Stewart JW, McGrath P, et al. Duloxetine vs placebo in treatment of chronic nonmajor depression. J Clin Psychiatry 2012; 73: 984-91. [PubMed: 22901348]

7. Hellerstein DJ, Stewart JW, Chen Y, Arunagiri V, Peterson BS, McGrath PJ. Desvenlafaxine vs placebo in the treatment of persistent depressive disorder. J Affect Disord 2019; 245: 403-11. [PubMed: 30423468]

8. Spitzer R, Williams J, Gibbon M. Structured clinical interview for DSM-IV (SCID). New York, NY: Biometrics Research, 1995.

9. Hamilton M. A rating scale for depression. J Neurol Neurosurg Psychiatry 1960; 23: 56-62. [PubMed: 14399272]

10. Whitfield-Gabrieli S, Nieto-Castanon A. Conn: a functional connectivity toolbox for correlated and anticorrelated brain networks. Brain Connect 2012; 2: 125-41. [PubMed: 22642651]

11. Whitfield-Gabrieli S, Ghosh SS, Nieto-Castanon A, et al. Brain connectomics predict response to treatment in social anxiety disorder. Mol Psychiatry 2016; 21: 680-85. [PubMed: 26260493]

12. Thompson WH, Thelin EP, Lilja A, Bellander BM, Fransson P. Functional resting-state fMRI connectivity correlates with serum levels of the S100B protein in the acute phase of traumatic brain injury. Neuroimage Clin 2016; 12: 1004-12. [PubMed: 27995066]

13. Beaty RE, Benedek M, Kaufman SB, Silvia PJ. Default and executive network coupling supports creative idea production. Sci Rep 2015; 5: 10964. [PubMed: 26084037]

14. Norman KA, Polyn SM, Detre GJ, Haxby JV. Beyond mind-reading: multi-voxel pattern analysis of fMRI data. Trends Cogn Sci 2006; 10(9): 424-30. [PubMed: 16899397]

15. Hair JF. Multivariate data analysis, 7th ed Upper Saddle River, NJ: Prentice Hall, 2010.

16. Wager TD, Atlas LY, Lindquist MA, Roy M, Woo CW, Kross E. An fMRI-based neurologic signature of physical pain. N Engl J Med 2013; 368: 1388-97. [PubMed: 23574118]

17. Smith SM, Fox PT, Miller KL, et al. Correspondence of the brain's functional architecture during activation and rest. Proc Natl Acad Sci USA 2009; 106: 13040-45. [PubMed: 19620724]

18. Posner J, Hellerstein DJ, Gat I, et al. Antidepressants normalize the default mode network in patients with dysthymia. JAMA Psychiatry 2013; 70: 373-82. [PubMed: 23389382]

19. VanderWeele T. A unification of mediation and interaction: a 4-way decomposition. Epidemiology 2014; 25: 749-61. [PubMed: 25000145]

20. Robins JM, Greenland S. Identifiability and exchangeability for direct and indirect effects. Epidemiology 1992; 3: 143-55. [PubMed: 1576220]

21. VanderWeele TJ, Vansteelandt S. Conceptual issues concerning mediation, interventions and composition. Stat Interface 2009; 2: 457-68.

22. Imai K, Keele L, Tingley D. A general approach to causal mediation analysis. Psychol Methods 2010; 15: 309. [PubMed: 20954780]

23. Li B, Liu L, Friston KJ, et al. A treatment-resistant default mode subnetwork in major depression. Biol Psychiatry 2013; 74: 48-54. [PubMed: 23273724]

24. Lisiecka D, Meisenzahl E, Scheuerecker J, et al. Neural correlates of treatment outcome in major depression. Int J Neuropsychopharmacol 2011; 14: 521-34. [PubMed: 21205435]

25. Anand A, Li Y, Wang Y, et al. Antidepressant effect on connectivity of the mood-regulating circuit: an fMRI study. Neuropsychopharmacol 2005; 30: 1334-44.

26. Hamilton JP, Furman DJ, Chang C, Thomason ME, Dennis E, Gotlib IH. Default-mode and taskpositive network activity in major depressive disorder: implications for adaptive and maladaptive rumination. Biol Psychiatry 2011; 70: 327-33. [PubMed: 21459364]

27. Posner J, Cha J, Wang Z, et al. Increased default mode network connectivity in individuals at high familial risk for depression. Neuropsychopharmacol 2015; 40: 1717-25. 
28. Gudayol-Ferré E, Peró-Cebollero M, González-Garrido AA, Guàrdia-Olmos J. Changes in brain connectivity related to the treatment of depression measured through fMRI: a systematic review. Front Hum Neurosci 2015; 9: 582. [PubMed: 26578927]

29. Horga G, Kaur T, Peterson BS. Annual research review: current limitations and future directions in MRI studies of child- and adult-onset developmental psychopathologies. J Child Psychol Psychiatry 2014; 55: 659-80. [PubMed: 24438507]

30. Demyttenaere K, Bonnewyn A, Bruffaerts R, Brugha T, De Graaf R, Alonso J. Comorbid painful physical symptoms and depression: prevalence, work loss, and help seeking. J Affect Disord 2006; 92: 185-93. [PubMed: 16516977]

31. Lopez-Sola M, Pujol J, Hernandez-Ribas R, et al. Effects of duloxetine treatment on brain response to painful stimulation in major depressive disorder. Neuropsychopharmacol 2010; 35: 2305-17.

32. Arnold LM, Meyers AL, Sunderajan P, et al. The effect of pain on outcomes in a trial of duloxetine treatment of major depressive disorder. Ann Clin Psychiatry 2008; 20: 187-93. [PubMed: 19034749]

33. Lindsay PG, Wyckoff M. The depression-pain syndrome and its response to antidepressants. Psychosomatics 1981; 22: 571-77. [PubMed: 7267947]

34. Smith GR. The epidemiology and treatment of depression when it coexists with somatoform disorders, somatization, or pain. Gen Hosp Psychiatry 1992; 14: 265-72. [PubMed: 1505748]

35. Chiu Y, Silman A, Macfarlane G, et al. Poor sleep and depression are independently associated with a reduced pain threshold. Results of a population based study. Pain 2005; 115: 316-21. [PubMed: 15911158]

36. Wang Y, Bernanke J, Peterson BS, Hellerstein DJ, Posner J. Antidepressant effects on the pain network in patients with depression The American College of Neuropsychopharmacology (ACNP) 56th Annual Meeting; Palm Springs, CA, USA; 12 3-7, 2017. 


\section{Research in context}

\section{Evidence before this study}

WHO considers depression the third leading cause of disability worldwide. Although antidepressant medications are effective for some, many patients either do not respond or have disruptive side-effects. Studies that combine neuroimaging with randomised clinical trials of antidepressants can identify mechanisms by which antidepressants exert their salutary effects, providing targets for new treatment development. To investigate the literature coupling neuroimaging with randomised controlled trials, we did a systematic search of PubMed up to April 1, 2019, with no language restrictions, using the search terms: "Magnetic Resonance Imaging" $\{\mathrm{MeSH}\}$ AND “Antidepressive Agents" $\{\mathrm{MeSH}\}$ AND "Clinical Trial" \{Publication Type $\}$. This yielded 147 studies. From these, we excluded studies that did not include participants with a depressive disorder, more than a single dose of the antidepressant agent, placebo control, or longitudinal functional neuroimaging. This exclusion resulted in three studies. The first included a sample of 32 patients with depression and the trial lasted 1 week only, the second included 25 patients and was placebo controlled for the first week only, and the third was our previous 10week, randomised, double-blind, placebo-controlled clinical trial of duloxetine.

\section{Added value of this study}

In two randomised controlled trials, larger and of longer duration than previous studies, we found that antidepressants significantly decreased functional connectivity compared with placebo within a well described thalamo-cortico-periaqueductal network that has previously been associated with the experience of pain. In the active treatment groups, the change in pain network density was correlated with reductions in depressive symptoms.

\section{Implications of all the available evidence}

Although depression and pain often co-occur, to our knowledge, this is the first placebocontrolled study to show the impact of antidepressants on the pain-related thalamocortico-periaqueductal network, also referred to as the "neurologic signature of physical pain". This consistent finding across two studies suggest a new and potentially important focus for the development of novel antidepressant therapeutics, as well as existing treatments, such as neurostimulation, which could target this neural system. 

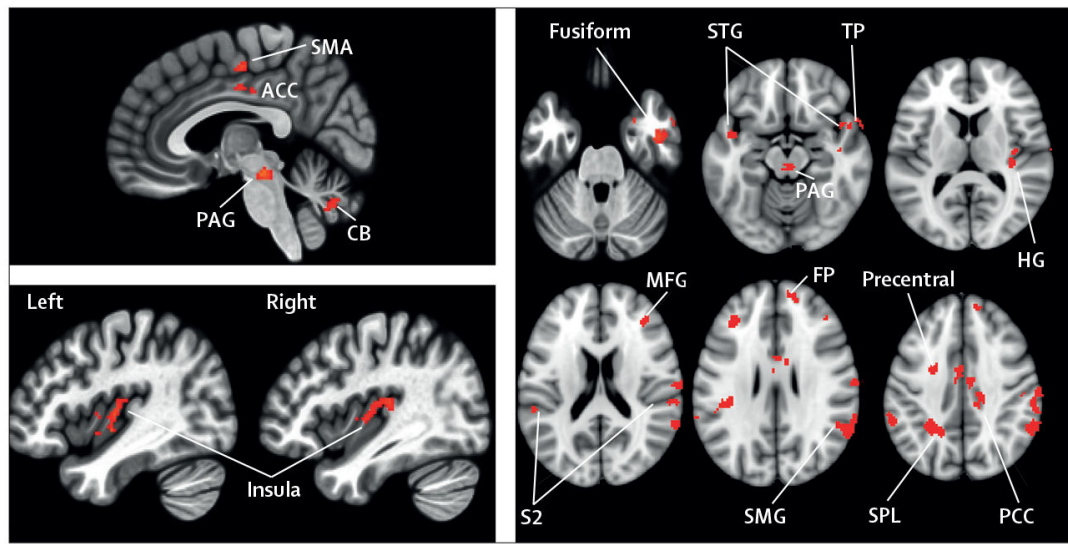

Figure 1: Multi-voxel pattern analysis-derived maps

Brain regions showing a significant treatment-by-time interaction in whole-brain connectivity from pooled data in two clinical trials ${ }^{6,7}$ of SNRI antidepressant medications. SMG = supramarginal gyrus. $\mathrm{SPL}=$ superior parietal lobule. midCC $=$ mid-cingulate cortex . $\mathrm{S} 2=$ secondary somatosensory cortex. $\mathrm{STG}=$ superior temporal gyrus. $\mathrm{MFG}=$ middle frontal gyrus. $\mathrm{PCC}=$ posterior cingulate gyrus. $\mathrm{PAG}=$ periaqueductal gray. $\mathrm{HG}=$ heschl's gyri. $\mathrm{TP}=$ temporal pole. $\mathrm{FP}=$ frontal pole. $\mathrm{SMA}=$ supplementary motor area. $\mathrm{CB}=$ cerebellum. 

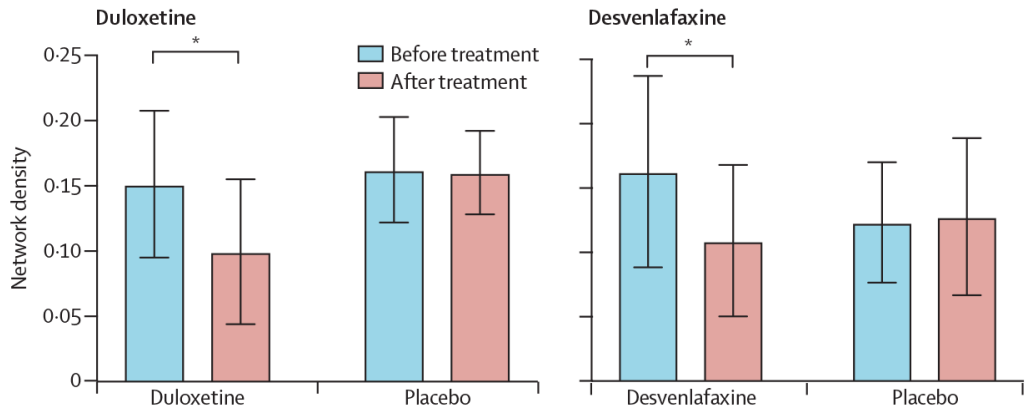

Figure 2: Pain network density before and after antidepressant treatment Error bars are SE. * $p<0.0001$. 

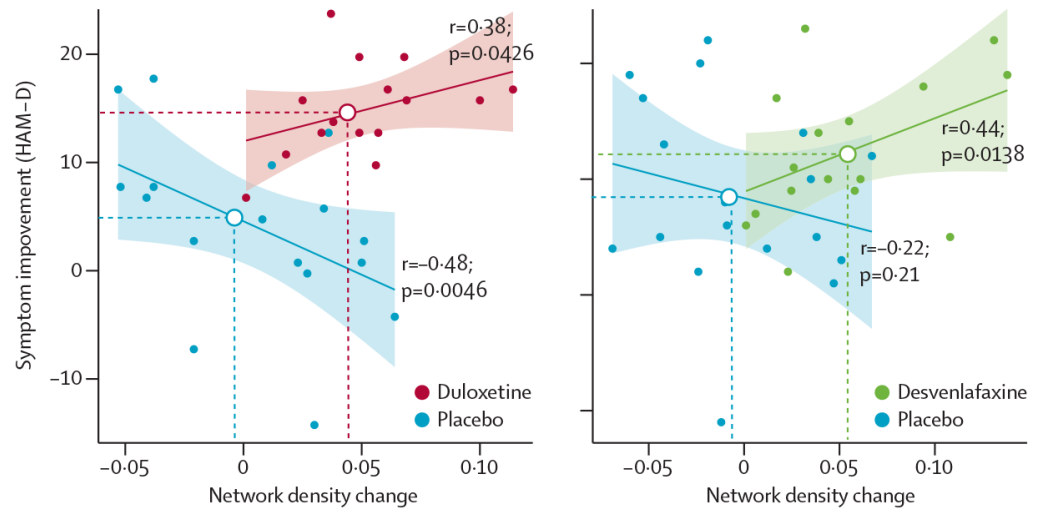

--O Mean value for network density and corresponding value of symptom improvement

Figure 3: Change in pain network density and depressive symptom severity Improvement in symptoms of depression was calculated as the difference between the 24item Hamilton Depression Rating Scale (HAM-D) summary score before versus after treatment (ie, positive values indicate improvement in symptoms). Change in pain network density was calculated as the difference in network density after versus before treatment (ie, a positive value denotes a decrease in density). Shaded area indicates $95 \% \mathrm{CI}$. 
Table 1:

Baseline characteristics of study participants

\begin{tabular}{|c|c|c|c|c|c|}
\hline & Total $(n=66)$ & Active treatment $(n=31)$ & Placebo $(n=35)$ & Test statistic & p value \\
\hline \multicolumn{6}{|l|}{ Demographic } \\
\hline Age, years & $37.27(11.86)$ & $38.77(12.61)$ & $35.94(11.16)$ & $\mathrm{t}=.0 .97$ & 0.34 \\
\hline Sex & . & . & . & $\chi^{2}=3.84$ & 0.05 \\
\hline Female & $32(48 \%)$ & $19(61 \%)$ & $13(37 \%)$ & . & . \\
\hline Male & $34(52 \%)$ & $12(39 \%)$ & $22(63 \%)$ & . & . \\
\hline Race or ethnicity & . & . & . & $\chi^{2}=5.52$ & 0.24 \\
\hline White & $44(67 \%)$ & $18(58 \%)$ & $26(74 \%)$ & . & . \\
\hline African American & $11(17 \%)$ & $5(16 \%)$ & $6(17 \%)$ & . & . \\
\hline Hispanic & $4(6 \%)$ & $4(13 \%)$ & 0 & . & . \\
\hline Asian & $5(8 \%)$ & $3(10 \%)$ & $2(6 \%)$ & . & . \\
\hline Not specified & $2(3 \%)$ & $1(3 \%)$ & $1(3 \%)$ & . & . \\
\hline \multicolumn{6}{|l|}{ Symptoms } \\
\hline HAM-D 24, pretreatment & $20.50(4.61)$ & $20.27(4.38)$ & $20.57(4.72)$ & $\mathrm{t}=-0.23$ & 0.82 \\
\hline HAM-D 17, pretreatment & $14.41(3.49)$ & $14.13(3.54)$ & $14.49(3.51)$ & $\mathrm{t}=-0.34$ & 0.73 \\
\hline Pain network density, ${ }^{*}$ pretreatment & $0.149(0.056)$ & $0.157(0.065)$ & $0.142(0.048)$ & $\mathrm{t}=1.07$ & 0.29 \\
\hline Age of onset of persistent depressive disorder, years & $15.15(9.70)$ & $12.47(6.12)$ & $15.94(10.44)$ & $\mathrm{t}=-1.61$ & 0.12 \\
\hline SCL-90-R pain & $1.37(1.76)$ & $1.50(1.83)$ & $1.34(1.76)$ & $\mathrm{t}=0.27$ & 0.79 \\
\hline SCL-90-R GSI & $1.00(0.49)$ & $0.89(0.42)$ & $1.03(0.51)$ & $\mathrm{t}=-0.97$ & 0.34 \\
\hline SCL-90-R anxiety & $0.78(0.58)$ & $0.58(0.41)$ & $0.83(0.60)$ & $\mathrm{t}=-1.65$ & 0.11 \\
\hline \multicolumn{6}{|l|}{ Comorbidities } \\
\hline Anxiety disorder & $15(23 \%)$ & $8(26 \%)$ & $7(20 \%)$ & . & . \\
\hline Previous substance use disorder & $14(21 \%)$ & $6(19 \%)$ & $8(23 \%)$ & . & . \\
\hline
\end{tabular}

Data are mean (SD) or $\mathrm{n}(\%)$. Due to rounding, total \% might be more than 100\%. HAM-D = 24-item and 17-item Hamilton Depression Rating Scale summary score. SCL-90-R = Symptom Checklist-90-Revisited; we defined SCL-90-R pain as the sum of two related items (appendix). GSI = global severity index.

Pain network density was identified and examined in the current study. 
Table 2:

Treatment effects identified by multivoxel pattern analysis

\begin{tabular}{llcll}
\hline & MNI coordinates $(\mathbf{x}, \mathbf{y}, \mathbf{z})$ & Cluster size & p-FDR & p-unc \\
\hline R AG, SMG, PoCG $^{*}$ & $(64,-48,22)$ & 963 & $<0.0001$ & $<0.00001$ \\
R TP, pInsula $^{*}$ & $(62,10,-14)$ & 282 & $<0.0001$ & $<0.00001$ \\
R pInsula $^{*}$ & $(40,-12,10)$ & 186 & 0.0007 & $<0.00001$ \\
L pInsula, STG $^{*}$ & $(-42,1,-11)$ & 175 & 0.0007 & $<0.00001$ \\
L SPL $^{*}$ & $(-24,-46,34)$ & 167 & 0.0009 & 0.00001 \\
midCC $^{*}$ & $(-4,-4,26)$ & 156 & 0.0010 & 0.00002 \\
R HG, pInsula $^{*}$ & $(34,-26,12)$ & 146 & 0.0012 & 0.00003 \\
L S2 $^{*}$ & $(-38,-28,28)$ & 112 & 0.0058 & 0.00015 \\
R STG $^{*}$ & $(46,2,-14)$ & 99 & 0.0093 & 0.00031 \\
L SMG, IPL $^{*}$ & $(-60,-42,40)$ & 95 & 0.0093 & 0.00038 \\
R fusiform, MTG & $(44,-8,-28)$ & 95 & 0.0093 & 0.00038 \\
Precentral gyrus & $(-26,-2,38)$ & 93 & 0.0093 & 0.00043 \\
PCC & $(16,-22,36)$ & 92 & 0.0093 & 0.00045 \\
Frontal pole & $(14,54,30)$ & 89 & 0.0098 & 0.00053 \\
R STG & $(60,-28,18)$ & 88 & 0.0099 & 0.00056 \\
R MFG & $(38,38,22)$ & 78 & 0.0298 & 0.00184 \\
PAG ${ }^{*}$ & $(0,-24,-14)$ & 68 & 0.0358 & 0.00386 \\
R cerebellum & $(20,-86,-42)$ & 0.0421 & 0.00422 \\
\hline
\end{tabular}

$\mathrm{MNI}=$ Montreal Neurological Institute. FDR $=$ false discovery rate. $u n c=$ uncorrected. $\mathrm{R}=$ right. $\mathrm{AG}=$ angular gyrus. $\mathrm{SMG}=$ supramarginal gyrus. $\mathrm{PoCG}=$ postcentral gyrus. $\mathrm{TP}=$ temporal pole. pInsula $=$ posterior insula $\mathrm{L}=$ left. $\mathrm{STG}=$ superior temporal gyrus. $\mathrm{SPL}=$ superior parietal lobule. midCC = mid-cingulate cortex. $\mathrm{HG}=$ Heschl's gyrus. $\mathrm{S} 2=$ secondary somatosensory cortex. IPL = inferior parietal lobule. MTG = middle temporal gyrus $. \mathrm{PCC}=$ posterior cingulate cortex $. \mathrm{MFG}=$ middle frontal gyrus. $\mathrm{PAG}=$ periaqueductal gray.

Clusters within the pain network. 16 